Supporting Information

\title{
Highly Selective Gas Sensors Based on Graphene Nanoribbons Grown by Chemical Vapor Deposition
}

\author{
Mikhail Shekhirev, ${ }^{1}$ Alexey Lipatov, ${ }^{1}$ Angel Torres, ${ }^{1}$ Nataliia S. Vorobeva, ${ }^{1}$ Ashley Harkleroad,${ }^{1}$ \\ Andrey Lashkov, ${ }^{2}$ Victor Sysoev, ${ }^{2,3}$ Alexander Sinitskii ${ }^{1,4 *}$ \\ ${ }^{1}$ Department of Chemistry, University of Nebraska - Lincoln, Lincoln, NE 68588, USA \\ ${ }^{2}$ Department of Physics, Yuri Gagarin State Technical University, Saratov, 410054, Russia \\ ${ }^{3}$ National University of Science and Technology “MISiS”, Moscow 119991, Russia \\ ${ }^{4}$ Nebraska Center for Materials and Nanoscience, University of Nebraska - Lincoln, Lincoln, NE \\ 68588, USA \\ *E-mail: sinitskii@unl.edu
}




\section{Supplementary Note 1. Raman spectra of GNRs synthesized in different conditions}
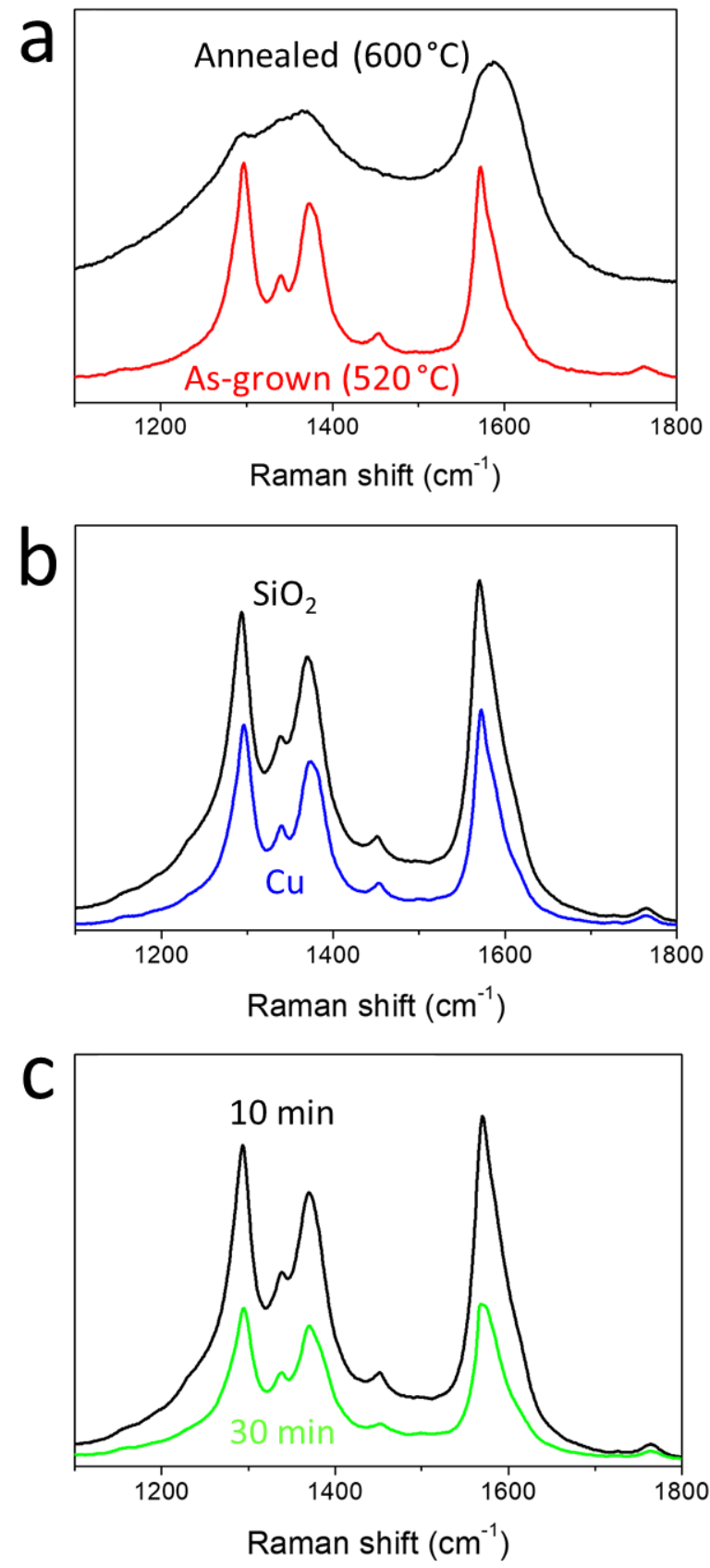

Figure S1. Raman spectra of GNRs synthesized in different conditions. (a) GNRs synthesized at $520{ }^{\circ} \mathrm{C}$ and the same film additionally annealed at $600{ }^{\circ} \mathrm{C}$ for $30 \mathrm{~min}$. (b) GNR films grown at $520{ }^{\circ} \mathrm{C}$ on $\mathrm{SiO}_{2}$ and $\mathrm{Cu}$ substrates. (c) $\mathrm{GNRs}$ synthesized on $\mathrm{Si} / \mathrm{SiO}_{2}$ at $520{ }^{\circ} \mathrm{C}$ for 10 and $30 \mathrm{~min}$. 


\section{Supplementary Note 2. Setup for gas sensing measurements}

The setup for gas sensing measurements is shown in Figure S2. We used an Agilent 4155C semiconductor parameter analyzer connected to a Keithley 7001 multiplexer to sequentially measure resistances of every individual sensing element on a chip. The chip was placed into a gas chamber $\left(V \sim 2 \mathrm{~cm}^{3}\right)$, which was kept at ambient temperature. An analyte was put in a vial with a custom-made horizontal capillary diffusion tube (Figure S2). If this diffusion vial is kept at a constant temperature, the concentration gradient of the analyte inside and outside the vial remains constant, which provides a constant driving force for the controlled release of an analyte. The bore diameter and the diffusion path length control the rate of release of a specific analyte from the diffusion vial. The Equation (1) was used to estimate the diffusion path length:

$$
L=1.9 \cdot 10^{4} \cdot T \cdot D \cdot M \cdot A \cdot \log \frac{P}{P-\rho} \cdot K \cdot \frac{1}{F \cdot C}
$$

where $L$ is the length of diffusion path $(\mathrm{cm}), T$ is the temperature of the vapor $(\mathrm{K}), D$ is the diffusion coefficient $\left(\mathrm{cm}^{2} / \mathrm{sec}\right)$ at $25^{\circ} \mathrm{C}$ and $1 \mathrm{~atm}, M$ is the molecular weight of an analyte $(\mathrm{g} / \mathrm{mol}), A$ is the cross-section area of the capillary $\left(\mathrm{cm}^{2}\right), P$ is the atmospheric pressure (mm Hg), $\rho$ is the vapor pressure of an analyte at the temperature $T(\mathrm{~mm} \mathrm{Hg}), K$ is the molar volume constant at $25^{\circ} \mathrm{C}$ and at $1 \mathrm{~atm}(K=24.47 / M), F$ is the total dilution flow $(\mathrm{sccm})$, and $C$ is the concentration, parts per million (ppm) by volume.

We used Line A to expose the GNR devices to an analyte and Line B to purge them with dry nitrogen as a background. Two independent mass flow controllers (MFC, Matheson Transducer, Model 8141) were used to maintain the same gas flow rates through both lines, and two flow switches were used to open and close these lines. When the GNR devices were purged 
with dry nitrogen through Line $\mathrm{B}$, the chamber with the diffusion vial was also continuously purged with dry nitrogen to prevent the accumulation of an analyte in the chamber.

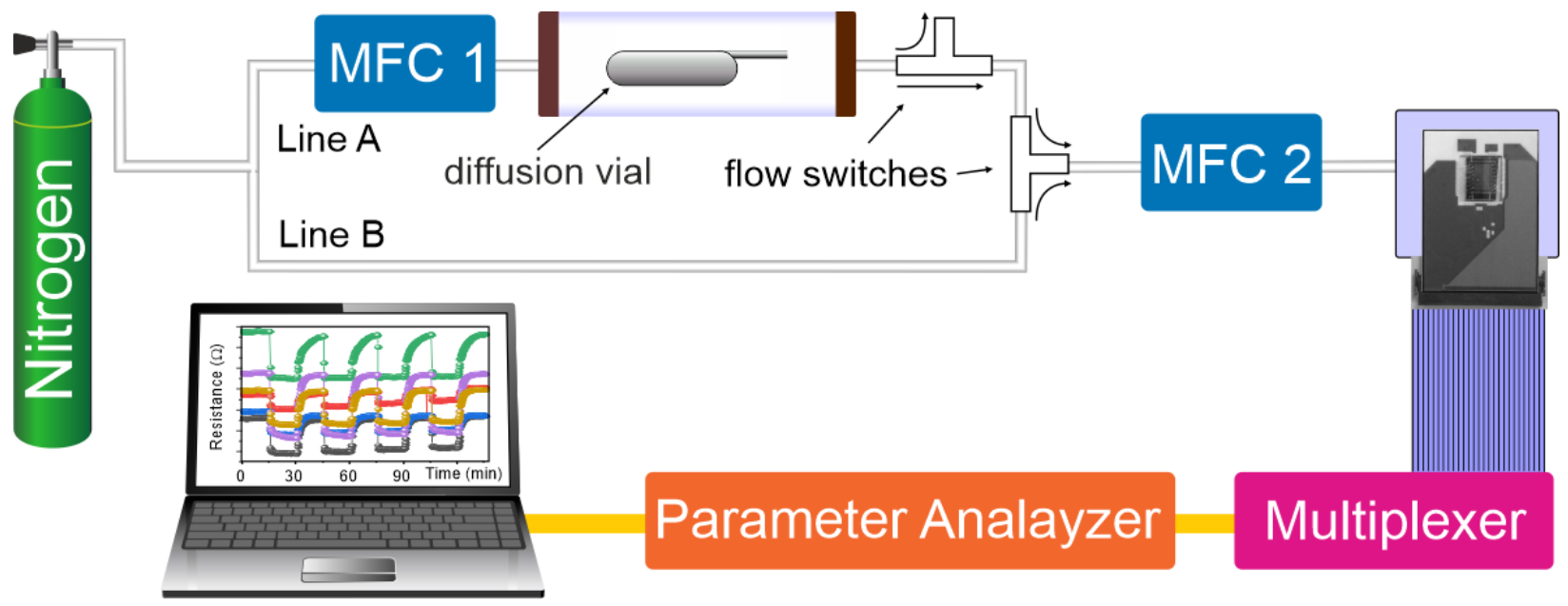

Figure S2. Experimental setup for sensor measurements. MFC - mass flow controller. 


\section{Supplementary Note 3. Additional sensor data}

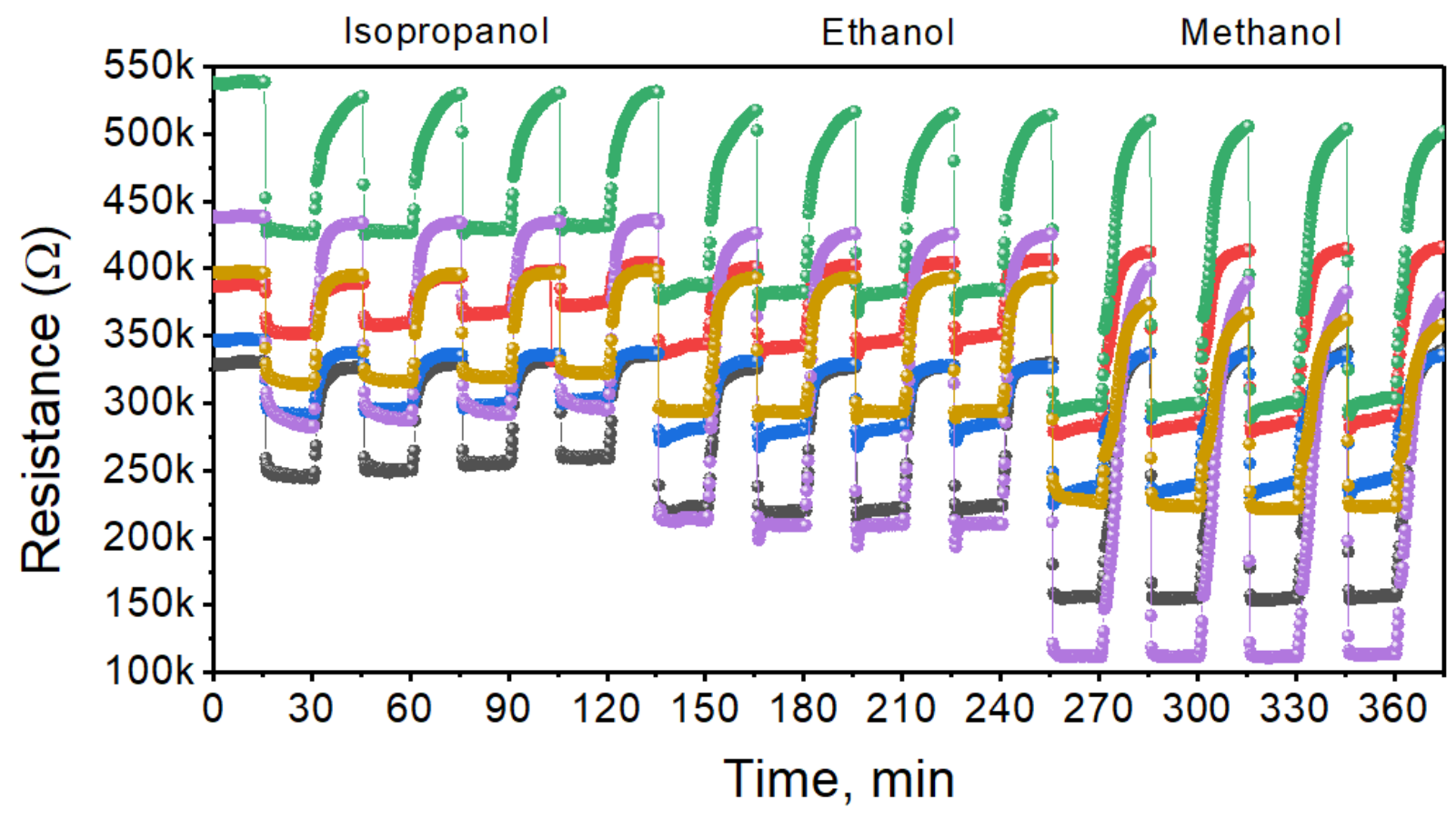

Figure S3. Responses of six different GNR-based sensor devices to isopropanol, ethanol and methanol mixed with dry $\mathrm{N}_{2}$ at concentrations of $500 \mathrm{ppm}$. 


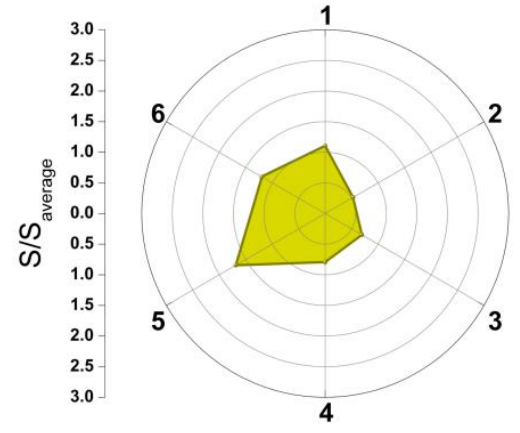

Triethylamine



Isopropanol

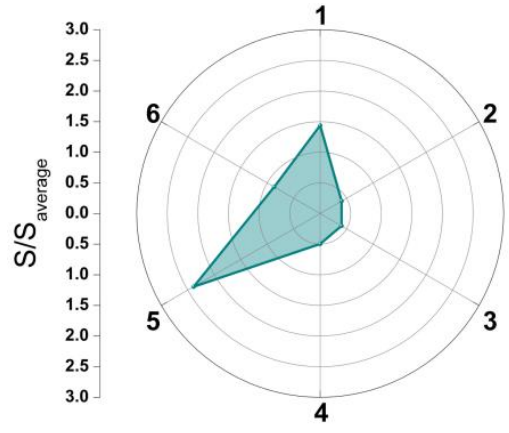

Diethylamine

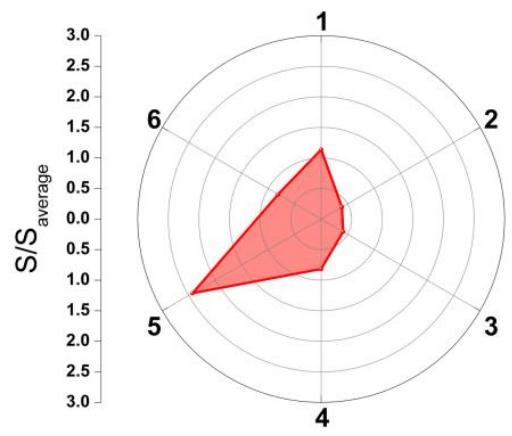

Ethanol

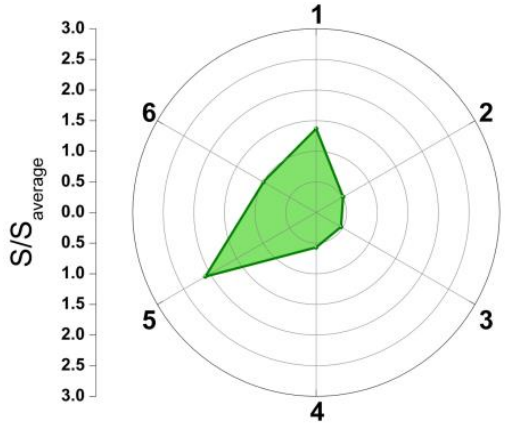

n-butylamine

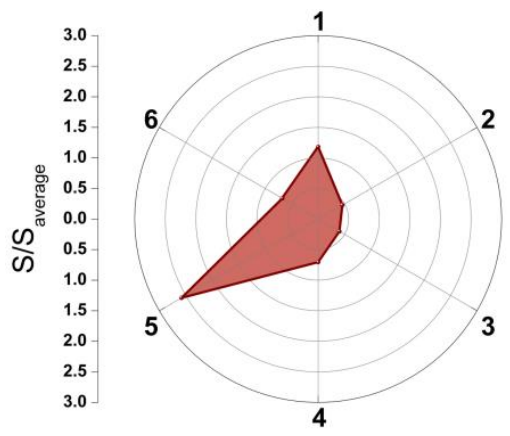

Methanol

Figure S4. Normalized responses of six sensors in the GNR-based multisensor array to three amine analytes (triethylamine, diethylamine and n-butylamine) and three alcohol analytes (isopropanol, ethanol and methanol). The raw sensor responses to analytes exhibited a large variation across the array. To improve the visual representation of sensor responses and demonstrate a "fingerprint" of each analyte we employed normalized values $(\mathrm{N})$, which were calculated as

$$
N=\frac{S_{i}}{S_{a v g}}
$$

where $S_{i}$ is a chemiresistive response of an individual sensor in the array and $S_{\text {avg }}$ is an average response across the array of $n$ sensors $(n=6)$ :

$$
S_{a v g}=\frac{\sum_{k=1}^{n} S_{k}}{n}
$$

